\title{
Synthesis and regioselective transformations of ethoxy-substituted 5-(perfluoroalkyl)pyrimidines
}

\author{
Mohamed A. Ismail,, ${ }^{a, b}$ Hanafi H. Zoorob, ${ }^{b}$ and Lucjan Strekowski, ${ }^{a,}$ \\ ${ }^{a}$ Department of Chemistry, Georgia State University, Atlanta, Georgia 30303, USA \\ ${ }^{b}$ Chemistry Department, Faculty of Science, Al-Mansoura University, Al-Mansoura, Egypt \\ E-mail: Lucian@gsu.edu
}

(received 01 Jul 2002; accepted 29 Aug 2002; published on the web 06 Sep 2002)

\begin{abstract}
The reaction of 5-bromo-2,4-diethoxypyrimidine or 5-bromo-2-ethoxypyrimidine with a perfluoroalkyl iodide in the presence of Cu-bronze followed by acid hydrolysis of the resultant 5perfluoroalkyl derivative furnishes the respective 5-perfluoroalkyluracil and 5perfluoroalkylpyrimidin-2(1H)one. The treatment of the perfluoroalkyluracil with $\mathrm{NaOH}$ or sodium alkoxide gives the respective 5-(perfluoroacyl)- or 5-(1,1-dialkoxyperfluoroalkyl)substituted uracil. 5-(Perfluoroalkyl)pyrimidine-2(1H)one is inert under these conditions.
\end{abstract}

Keywords: Pyrimidines, uracils, perfluoroalkylation, hydrolysis, regioselective transformations

\section{Introduction}

Fluorine-containing derivatives of pyrimidines including uracils and nucleosides are potent antitumor and antiviral agents. ${ }^{1-4}$ Most previous synthetic work has focused on the preparation of 5-(trifluoromethyl)uracil and its nucleosides, and reports on the synthesis of higher perfluoroalkyl derivatives are scarce. The reported synthetic routes to such compounds are inefficient, require the use of specialized equipment and starting materials that are difficult to obtain, and/or utilize toxic reagents or solvents. These are perfluoroalkylation of uracil and uridine by the reaction of their bis(trimethylsilyl) derivatives with bis(perfluoroalkanoyl) peroxides, ${ }^{5}$ photochemically induced coupling by the reaction of uracil, its nucleosides or their expensive 5-iodo derivatives with toxic bis(perfluoroalkyl)mercury, ${ }^{6}$ the electrochemically induced coupling, ${ }^{7,8}$ and the highly inefficient reaction of uracil, its nucleosides or 5-iodouracil with a perfluoroalkyl iodide in the presence of copper bronze. ${ }^{9,10}$ The quite efficient reaction of 5-iodouracil nucleosides with trifluoromethylcopper requires an elaborate purification of the copper reagent but, in principle, could be extended to the preparation of higher perfluoroalkyl 
analogs. ${ }^{11}$ Unfortunately, this synthesis is not acceptable because the reaction, as reported, must be conducted in hexamethylphosphoramide which is a highly potent carcinogen.

Since 5-(perfluoroalkyl)uracils can be efficiently transformed into nucleosides, ${ }^{3,4}$ our work has been focused on finding a facile synthesis of the former compounds, ${ }^{12}$ as described in this paper. We also report efficient chemical modifications of 5-(perfluoroalkyl)uracils that involve regioselectively the benzylic-type difluoromethylene moiety of the perfluoroalkyl substituent.

\section{Results and Discussion}

In contrast to the inefficient coupling reaction of a 5-halouracil with a perfluoroalkyl iodide, as mentioned above, ${ }^{9,10}$ the treatment of 5-bromo-2,4-diethoxypyrimidine (1) with perfluorobutyl iodide or perfluorohexyl iodide in the presence of activated copper bronze ${ }^{9}$ in DMSO gave the respective 5-(perfluoroalkyl)pyrimidines 2a,b in high yields (Scheme 1). Acid-mediated hydrolysis of $\mathbf{2}$ furnished 5-(perfluoroalkyl)uracils 3, also in high yields. Compounds 3a,b are stable under acidic conditions but undergo an efficient hydrolysis by the reaction with hydroxide ion in aqueous solution to give the respective 5-(perfluoroacyl)uracils $\mathbf{6 a} \mathbf{a}, \mathbf{b}$.

The suggested mechanism for $\mathbf{6}$ (Scheme 1) involves the intermediacy of $\mathbf{4}$ and $\mathbf{5}$ with a subsequent generation of similar derivatives and is consistent with the general pattern of the chemistry of the anionically activated perfluoroalkyl group in 2-perfluoroalkyl and 4perfluoroalkyl-substituted anilines. ${ }^{13-16}$ A related mechanistic pathway has also been suggested for inhibition of thymidylate syntheses by 5-(trifluoromethyl)uracil which is used as an antitumor drug. 17
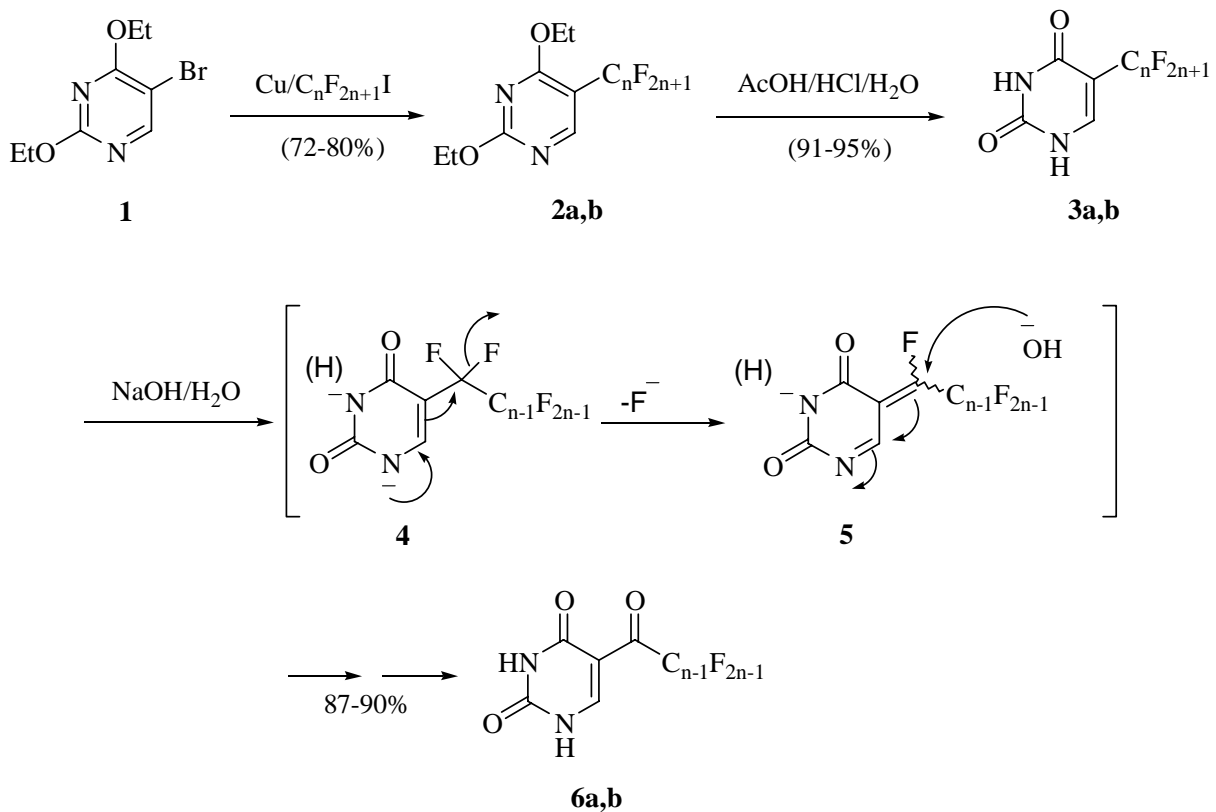

Scheme 1. a: $n=4 ; \mathbf{b}: n=6$. 
Although perfluoroalkyl ketones, including 6, resist the formation of $\mathrm{sp}^{2}$-type condensation products such as hydrazones, they readily undergo an addition reaction with a number of nucleophiles to form stable $\mathrm{sp}^{3}$-type adducts. ${ }^{18}$ A greatly simplified synthetic route to acetals 7-9 by the reaction of 5-(perfluoroalkyl)pyrimidines $\mathbf{3}$ with alkoxide ions is given in Scheme 2 . Interesting features in the ${ }^{1} \mathrm{H}$ NMR spectra of these acetals are a broadened signal for $O$ methylene protons of the ethoxy derivatives 7 and a well-defined single AB absorption pattern for the $O$-methylene protons of the benzyloxy groups of $\mathbf{8}$. As shown by decoupling experiments, these unusual absorption patterns are not due to a proton-fluorine coupling and, therefore, they must be a result of restricted rotation of the alkoxy groups around the central carbon atom of the acetal. In particular, the ${ }^{1} \mathrm{H}$ NMR spectra of $\mathbf{8}$ are consistent with the presence of a symmetric equilibrium conformation in which the pyrimidine plane bisects the acetal functionality. Due to symmetry, the two methylene moieties are equivalent but their geminal protons are not, thereby giving rise to a single $\mathrm{AB}$ system in the ${ }^{1} \mathrm{H}$ NMR spectrum, as observed. An interesting feature in the ${ }^{1} \mathrm{H}$ NMR spectrum of acetal 9 is a long-range coupling between $\mathrm{C6}-\mathrm{H}$ of the uracil and two fluorine atoms of the perfluoroalkyl chain. This coupling gives rise to a doublet of doublets for C6-H at $\delta 7.56$.<smiles>[Y7]CCC([R20])(O[R])c1c[nH]c(=O)[nH]c1=O</smiles>

7a,b: $\mathrm{R}=\mathrm{C}_{2} \mathrm{H}_{5}$

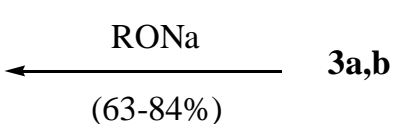

$(63-84 \%)$

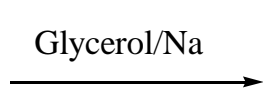

$(51 \%)$

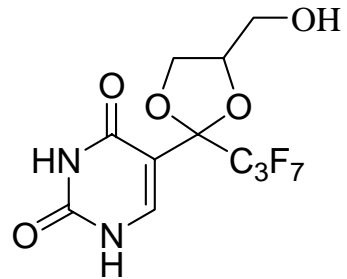

9

8a,b: $\mathrm{R}=\mathrm{C}_{6} \mathrm{H}_{5} \mathrm{CH}_{2}$

Scheme 2. $\mathbf{a}: \mathrm{n}=3 ; \mathbf{b}: \mathrm{n}=5$.

An extension of this work on the successful synthesis of 2-ethoxy-5(perfluoroalkyl)pyrimidines 11a,b, starting with 5-bromo-2-ethoxypyrimidine (10) and using a similar methodology, is given in Scheme 3. These products were efficiently hydrolyzed to give the expected 5-(perfluoroalkyl)pyrimidine-2(1H)ones 12a,b. In contrast to the facile transformations of their uracil analogs 3, as discussed above, compounds $\mathbf{1 2}$ are inert in the presence of base, even under forced conditions of an elevated temperature. For example, pyrimidinone 12a was recovered in a virtually quantitative yield after its solution in aqueous sodium hydroxide had been heated in a pressure vessel to $120^{\circ} \mathrm{C}$ for $4 \mathrm{~h}$. 

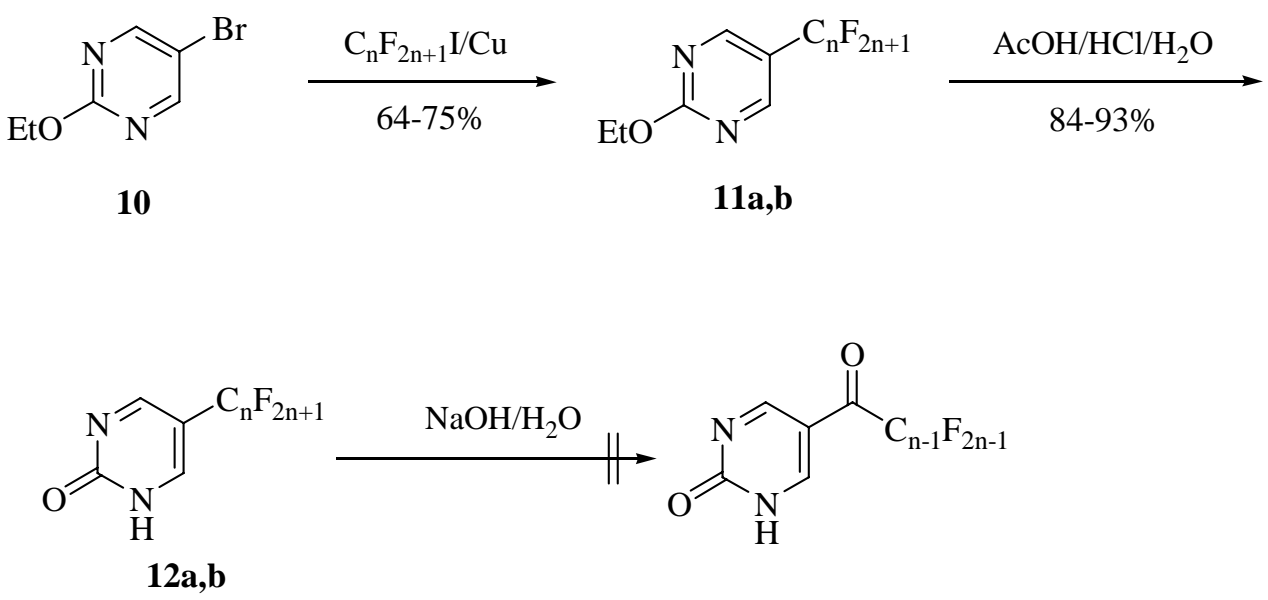

Scheme 3. a: $n=4 ; \mathbf{b}: n=6$.

\section{Experimental Section}

General Procedures. Melting points (Pyrex capillary) are not corrected. ${ }^{1} \mathrm{H}$ NMR (400 MHz) and ${ }^{19} \mathrm{~F}$ NMR (282 MHz) spectra were recorded in $\mathrm{CDCl}_{3}$ at $25{ }^{\circ} \mathrm{C}$ or DMSO- $d_{6}$ at $30{ }^{\circ} \mathrm{C}$ with TMS and $\mathrm{C}_{6} \mathrm{~F}_{6}$ as the respective internal standards. Crude mixtures were analyzed, and EI mass spectra of pure components were obtained on a GC-MS instrument equipped with an on-column injector, a poly(dimethylsiloxane)-coated capillary column, and a mass selective detector operating at $70 \mathrm{eV}$.

\section{2,4-Diethoxy-5-(perfluoroalkyl)pyrimidines 2a,b}

5-Bromo-2,4-dichloro-pyrimidine was allowed to react with sodium ethoxide in ethanol as described previously ${ }^{19}$ and resultant 5-bromo-2,4-diethoxypyrimidine (1) was isolated by using the following simplified procedure. Thus, concentration of the mixture followed by extraction of 1 from the residue with ether/pentanes (3:1) and then concentration of the extract and cooling the residual solution resulted in crystallization of 1 : yield $92 \%$, mp $69-70{ }^{\circ} \mathrm{C}$ (reported ${ }^{19} \mathrm{mp} 69{ }^{\circ} \mathrm{C}$ ).

A mixture of a perfluoroalkyl iodide $(12 \mathrm{mmol}), \mathbf{1}(2.0 \mathrm{~g}, 8 \mathrm{mmol})$, Cu-bronze ${ }^{9}$ (1.7 g, $27 \mathrm{mmol})$, and anhydrous DMSO $(8 \mathrm{~mL})$ was stirred under a nitrogen atmosphere and heated to $110{ }^{\circ} \mathrm{C}$ for $20 \mathrm{~h}$ in a flask equipped with a dry-ice condenser. After cooling the mixture was poured into ether $(200 \mathrm{~mL})$ and filtered. The solution was washed with water $(25 \mathrm{~mL})$, dried $\left(\mathrm{MgSO}_{4}\right)$, and concentrated. Compounds 2 were obtained by flash chromatography on silica gel (30 g) by eluting with hexanes/AcOEt (19:1) followed by crystallization from ether/hexanes (2:1).

2,4-Diethoxy-5-(perfluorobutyl)pyrimidine (2a). Yield 72\%; mp 35-36 ${ }^{\circ} \mathrm{C} ;{ }^{1} \mathrm{H}$ NMR $\left(\mathrm{CDCl}_{3}\right)$ $\delta 1.42(\mathrm{~m}, 6 \mathrm{H}), 4.48(\mathrm{~m}, 4 \mathrm{H}), 8.40(\mathrm{~s}, 1 \mathrm{H}) ;{ }^{19} \mathrm{~F} \mathrm{NMR}\left(\mathrm{CDCl}_{3}\right) \delta 35.7$ (2F), $39.2(2 \mathrm{~F}), 52.1(2 \mathrm{~F})$, 
80.8 (3F); EI-MS m/z 161 (100), $386\left(10, \mathrm{M}^{+}\right.$). Analysis. Calcd. for $\mathrm{C}_{12} \mathrm{H}_{11} \mathrm{~F}_{9} \mathrm{~N}_{2} \mathrm{O}_{2}$ : C, 37.32; $\mathrm{H}$, 2.87; N, 7.25. Found: C, 37.56; H, 2.78; N, 7.16.

2,4-Diethoxy-5-(perfluorohexyl)pyrimidine (2b). Yield 80\%; mp 50-51 ${ }^{\circ} \mathrm{C} ;{ }^{1} \mathrm{H} \mathrm{NMR}\left(\mathrm{CDCl}_{3}\right)$ $\delta 1.42(\mathrm{~m}, 6 \mathrm{H}), 4.47$ (m, 4H), $8.40(\mathrm{~s}, 1 \mathrm{H}) ;{ }^{19} \mathrm{~F} \mathrm{NMR}\left(\mathrm{CDCl}_{3}\right) \delta 35.4(2 \mathrm{~F}), 38.8(2 \mathrm{~F}), 39.7$ (2F), 40.0 (2F), 52.0 (2F), 80.3 (3F); EI-MS m/z 161 (100), 486 (20, $\mathrm{M}^{+}$). Analysis. Calcd. for $\mathrm{C}_{14} \mathrm{H}_{11} \mathrm{~F}_{13} \mathrm{~N}_{2} \mathrm{O}_{2}$ : C, 34.58; H, 2.28; N, 5.76. Found: C, 34.42; H, 2.32; N, 5.68.

\section{5-(Perfluoroalkyl)uracils 3a,b}

A solution prepared from 2 (5 mmol), acetic acid $(25 \mathrm{~mL})$, and hydrochloric acid (2N, $5 \mathrm{ml})$ was heated under reflux for $1 \mathrm{~h}$. Concentration under a reduced pressure followed by trituration of the crystalline residue with ether gave uracil 3.

5-(Perfluorobutyl)uracil (3a). Yield 91\%; mp 270-272 ${ }^{\circ} \mathrm{C}$ (reported ${ }^{9} \mathrm{mp}>250{ }^{\circ} \mathrm{C}$ ); ${ }^{1} \mathrm{H}$ NMR $\left(\mathrm{DMSO}-d_{6}\right) \delta 8.00$ (s, $\left.1 \mathrm{H}\right), 11.6$ (bs, exchangeable with $\left.\mathrm{D}_{2} \mathrm{O}, 2 \mathrm{H}\right) ;{ }^{19} \mathrm{~F}$ NMR (DMSO- $\left.d_{6}\right) \delta 37.0$ (2F), 41.0 (2F), 54.0 (2F), 82.0 (3F); EI-MS m/z 161 (100), 330 (10, $\mathrm{M}^{+}$).

5-(Perfluorohexyl)uracil (3b). Yield 95\%; mp 279-281 ${ }^{\circ} \mathrm{C}$, decomp. (reported ${ }^{5} \mathrm{mp}$ 277-278 ${ }^{\circ} \mathrm{C}$, decomp.); ${ }^{1} \mathrm{H}$ NMR (DMSO- $\left.d_{6}\right) \delta 8.00$ (s, $\left.1 \mathrm{H}\right), 11.6$ (bs, exchangeable with $\mathrm{D}_{2} \mathrm{O}, 2 \mathrm{H}$ ); ${ }^{19} \mathrm{~F}$ NMR (DMSO-d $\left.)_{6}\right) 37.0$ (2F), 40.0 (2F), 41.0 (2F), 42.0 (2F), 54.1 (2F), 82.0 (3F); EI-MS m/z 161 (100), $430\left(10, \mathrm{M}^{+}\right)$.

\section{5-(Perfluoroacyl)uracils 6a,b}

A solution of $3(1 \mathrm{mmol})$ in aqueous $\mathrm{NaOH}(0.2 \mathrm{M}, 25 \mathrm{~mL})$ was heated to $65{ }^{\circ} \mathrm{C}$ for $2 \mathrm{~h}$, and then was acidified with hydrochloric acid $(1 \mathrm{M})$ and extracted with ethyl acetate $(3 \times 25 \mathrm{~mL})$. The extract was dried $\left(\mathrm{MgSO}_{4}\right)$ and concentrated, and the resultant solid was crystallized from EtOH (6a) or $\mathrm{MeOH}(\mathbf{6 b})$.

5-(Perfluorobutanoyl)uracil (6a). Yield 87\%; mp 274-278 ${ }^{\circ} \mathrm{C}$, decomp.; ${ }^{1} \mathrm{H}$ NMR (DMSO- $d_{6}$ ) $\delta 8.31$ (s, $1 \mathrm{H}$ ), 11.6 (bs, exchangeable with $\mathrm{D}_{2} \mathrm{O}, 1 \mathrm{H}$ ), 12.2 (bs, exchangeable with $\mathrm{D}_{2} \mathrm{O}, 1 \mathrm{H}$ ); ${ }^{19} \mathrm{~F}$ NMR (DMSO-d $\left.)_{6}\right) \delta 38.4(2 \mathrm{~F}), 48.1$ (2F), 82.8 (3F). Analysis. Calcd. for $\mathrm{C}_{8} \mathrm{H}_{3} \mathrm{~F}_{7} \mathrm{~N}_{2} \mathrm{O}_{3}: \mathrm{C}, 31.19$; H, 0.98; N, 9.09. Found: C, 31.35; H, 1.15; N, 8.84.

5-(Perfluorohexanoyl)uracil (6b). Yield 90\%; mp 287-290 ${ }^{\circ} \mathrm{C}$, decomp.; ${ }^{1} \mathrm{H}$ NMR (DMSO- $d_{6}$ ) $\delta 8.31$ (s, 1H), 11.6 (bs, exchangeable with $\mathrm{D}_{2} \mathrm{O}, 1 \mathrm{H}$ ), 12.2 (bs, exchangeable with $\mathrm{D}_{2} \mathrm{O}, 1 \mathrm{H}$ ); ${ }^{19} \mathrm{~F}$ NMR (DMSO-d $)_{6} \delta 36.7$ (2F), 41.0 (2F), 42.5 (2F), 48.6 (2F), 82.3 (3F). Analysis. Calcd. for $\mathrm{C}_{10} \mathrm{H}_{3} \mathrm{~F}_{11} \mathrm{~N}_{2} \mathrm{O}_{3}$ : C, 29.41; H, 0.74; N, 6.86. Found: C, 29.15; H, 0.99; N, 6.62.

\section{Acetals 7a,b}

A solution of $3(1 \mathrm{mmol})$ in absolute $\mathrm{EtOH}(4 \mathrm{~mL})$ was added under a nitrogen atmosphere to a solution of sodium ethoxide prepared from sodium (115 mg, $5 \mathrm{mmol}$ ) and absolute $\mathrm{EtOH}$ $(5 \mathrm{~mL})$. The mixture was heated under reflux for $8 \mathrm{~h}$ and then concentrated. Water $(6 \mathrm{~mL})$ was added to the residue and the solution was neutralized with Dowex cation exchange resin or hydrochloric acid ( $1 \mathrm{M}$ ) and concentrated. The product was crystallized from EtOH.

5-(1,1-Diethoxyperfluorobutyl)uracil (7a). Yield 79\%; mp 242-243 ${ }^{\circ} \mathrm{C}$; ${ }^{1} \mathrm{H}$ NMR (DMSO- $\left.d_{6}\right) \delta$ $1.16(\mathrm{t}, \mathrm{J}=7 \mathrm{~Hz}, 6 \mathrm{H}), 3.58(\mathrm{~m}, 4 \mathrm{H}), 7.60$ (s, 1H), 11.2 (bs, exchangeable with $\left.\mathrm{D}_{2} \mathrm{O}, 2 \mathrm{H}\right) ;{ }^{19} \mathrm{~F}$ 
NMR (DMSO-d ) $\delta 38.6$ (2F), 46.0 (2F), 82.5 (3F); EI-MS m/z 139 (100), 383 (20, M $\left.^{+}+1\right)$. Analysis. Calcd. for $\mathrm{C}_{12} \mathrm{H}_{13} \mathrm{~F}_{7} \mathrm{~N}_{2} \mathrm{O}_{4}$ : C, 37.71; H, 3.43; N, 7.33. Found: C, 37.64; H, 3.47; N, 7.16.

5-(1,1-Diethoxyperfluorohexyl)uracil (7b). Yield 84\%; mp 245-246 ${ }^{\circ} \mathrm{C}$; ${ }^{1} \mathrm{H}$ NMR (DMSO- $d_{6}$ ) $\delta 1.16$ (t, J = $7 \mathrm{~Hz}, 6 \mathrm{H}), 3.56(\mathrm{~m}, 4 \mathrm{H}), 7.56$ (s, 1H), 11.2 (bs, exchangeable with $\left.\mathrm{D}_{2} \mathrm{O}, 2 \mathrm{H}\right) ;{ }^{19} \mathrm{~F}$ NMR (DMSO- $\left.d_{6}\right) \delta 36.8$ (2F), 40.5 (2F), 42.4 (2F), 46.7 (2F), 82.3 (3F); EI-MS m/z 139 (100), $483\left(25, \mathrm{M}^{+}+1\right)$. Analysis. Calcd. for $\mathrm{C}_{14} \mathrm{H}_{13} \mathrm{~F}_{11} \mathrm{~N}_{2} \mathrm{O}_{4}$ : C, 34.87; H, 2.72; N, 5.81. Found: C, 35.02; H, 2.76; N, 5.77.

\section{Acetals 8a,b}

Sodium benzyloxide prepared from sodium hydride $(120 \mathrm{mg}, 5 \mathrm{mmol})$ and benzyl alcohol (5 mL) was allowed to react with $3(1 \mathrm{mmol})$ in benzyl alcohol $(5 \mathrm{~mL})$ and the mixture was worked up as described above. The product was crystallized from ether.

5-(1,1-Dibenzyloxyperfluorobutyl)uracil (8a). Yield 66\%; mp 225-227 ${ }^{\circ} \mathrm{C}$; ${ }^{1} \mathrm{H}$ NMR (DMSO$\left.d_{6}\right) \delta 4.63$ (d, J = 11.5 Hz, 2H), 4.77 (d, J = 11.5 Hz, 2H), 7.36 (m, 10H), 7.65 (s, 1H), 11.3 (bs, exchangeable with $\mathrm{D}_{2} \mathrm{O}, 2 \mathrm{H}$ ); ${ }^{19} \mathrm{~F}$ NMR (DMSO-d $\left.)_{6}\right) \quad 38.6$ (2F), 46.4 (2F), 82.5 (3F); EI-MS m/z 308 (100), 507 (10, $\mathrm{M}^{+}+1$ ). Analysis. Calcd. for $\mathrm{C}_{22} \mathrm{H}_{17} \mathrm{~F}_{7} \mathrm{~N}_{2} \mathrm{O}_{4}$ : C, 52.18; H, 3.38; N, 5.53. Found: C, 52.46; H, 3.33; N, 5.57.

5-(1,1-Dibenzyloxyperfluorohexyl)uracil (8b). Yield 63\%; mp 224-225 ${ }^{\circ} \mathrm{C} ;{ }^{1} \mathrm{H}$ NMR (DMSO$\left.d_{6}\right) \delta 4.62$ (d, J = 11.5 Hz, 2H), 4.76 (d, J = 11.5 Hz, 2H), 7.36 (m, 10H), 7.66 (s, 1H), 11.3 (bs, exchangeable with $\left.\mathrm{D}_{2} \mathrm{O}, 2 \mathrm{H}\right) ;{ }^{19} \mathrm{~F}$ NMR (DMSO- $d_{6}$ ) $\delta 36.9$ (2F), 40.6 (2F), 42.5 (2F), 47.1 (2F), 82.3 (3F). Analysis. Calcd. for $\mathrm{C}_{24} \mathrm{H}_{17} \mathrm{~F}_{11} \mathrm{~N}_{2} \mathrm{O}_{4}$ : C, 47.54; H, 2.83; N, 4.62. Found: C, 47.69; $\mathrm{H}$, 2.86; N, 4.64.

5-(2-Heptafluoropropyl-4-hydroxymethyl-1,3-dioxolan-2-yl)uracil (9). A mixture of glycerol $(5 \mathrm{~mL})$ and metallic sodium $\left(0.115 \mathrm{~g}, 5 \mathrm{mmol}\right.$ ) was stirred at $23{ }^{\circ} \mathrm{C}$ until evolution of hydrogen ceased and then treated with a solution of 3a $(0.33 \mathrm{~g}, 1 \mathrm{mmol})$. The mixture was heated to $150{ }^{\circ} \mathrm{C}$ for $8 \mathrm{~h}$, concentrated by distillation of glycerol on a Kugelrohr, and the residue was dissolved in water $(6 \mathrm{~mL})$. The solution was neutralized with hydrochloric acid $(1 \mathrm{M})$ and concentrated on a rotary evaporator to $4 \mathrm{~mL}$. The resultant precipitate of $\mathbf{9}$ was filtered and crystallized from EtOH: yield 0.195g (51\%); mp 273-274 ${ }^{\circ} \mathrm{C} ;{ }^{1} \mathrm{H}$ NMR (DMSO-d $\left.{ }_{6}\right) \delta 3.50$ (m, 2H), $3.80(\mathrm{~m}, 1 \mathrm{H}), 4.24$ (m, 2H), 5.01(bs, exchangeable with $\left.\mathrm{D}_{2} \mathrm{O}, 1 \mathrm{H}\right), 7.56\left(\mathrm{dd}, \mathrm{J}_{\mathrm{H}-\mathrm{F}}=29 \mathrm{~Hz}, \mathrm{~J}_{\mathrm{H}-\mathrm{F}}=6 \mathrm{~Hz}, 1 \mathrm{H}\right) 11.20$ (bs, exchangeable with $\mathrm{D}_{2} \mathrm{O}, 2 \mathrm{H}$ ); ${ }^{19} \mathrm{~F}$ NMR (DMSO- $\left.d_{6}\right) \delta 38.2$ (2F), $44.3(2 \mathrm{~F}), 82.2(3 \mathrm{~F})$; ); FABMS (thioglycerol) $\mathrm{m} / \mathrm{z} 383\left(100, \mathrm{M}^{+}+1\right)$, 308 (60). Analysis. Calcd. for $\mathrm{C}_{11} \mathrm{H}_{9} \mathrm{~F}_{7} \mathrm{~N}_{2} \mathrm{O}_{5}$ : C, 34.56; H, 2.37; N, 7.32. Found: C, 34.76; H, 2.37; N, 7.25.

\section{2-Ethoxy-5-(perfluoroalkyl)pyrimidines 11a,b}

5-Bromopyrimidin-2(1H)one was obtained by the following modification of the published procedure. ${ }^{20}$ Pyrimidin-2(1H)one hydrochloride $(5.0 \mathrm{~g}, 38 \mathrm{mmol})$ was added gradually at $23{ }^{\circ} \mathrm{C}$ to a stirred solution of bromine $(9.0 \mathrm{~g}, 56 \mathrm{mmol})$ in water $(100 \mathrm{~mL})$ and then the mixture was 
stirred at $50{ }^{\circ} \mathrm{C}$ for $3 \mathrm{~h}$. Concentration under a reduced pressure followed by crystallization of the residue from 95\% EtOH gave 5-bromopyrimidin-2(1H)one: yield 88\%, mp 233-235 ${ }^{\circ} \mathrm{C}$ (reported ${ }^{20}$ yield $55 \%$, mp $234-235{ }^{\circ} \mathrm{C}$ ). Reaction of this product with $\mathrm{POCl}_{3}{ }^{21}$ followed by the reaction of the resultant 5-bromo-2-chloropyrimidine with sodium ethoxide ${ }^{22}$ according to the published procedures furnished 5-bromo-2-ethoxypyrimidine (10). Compounds 11a,b were synthesized by treatment of $\mathbf{1 0}$ with a perfluoroalkyl iodide in the presence of copper bronze as described above for 2 and purified by flash chromatography on silica gel (pentanes/ether, 4:1). Compound 11b was crystallized from ether.

2-Ethoxy-5-(perfluorobutyl)pyrimidine (11a). Yield 64\%; an oil; ${ }^{1} \mathrm{H}$ NMR $\left(\mathrm{CDCl}_{3}\right) \delta 1.47$ (t, J $=7 \mathrm{~Hz}, 3 \mathrm{H}), 4.53(\mathrm{q}, \mathrm{J}=7 \mathrm{~Hz}, 2 \mathrm{H}), 8.70(\mathrm{~s}, 2 \mathrm{H}) ;{ }^{19} \mathrm{~F} \mathrm{NMR}\left(\mathrm{CDCl}_{3}\right) \delta 36.3(2 \mathrm{~F}), 39.0(2 \mathrm{~F}), 50.4$ (2F), 80.8 (3F). EI-MS m/z 145 (100), 342 (25, $\mathrm{M}^{+}$). Analysis. Calcd. for $\mathrm{C}_{10} \mathrm{H}_{7} \mathrm{~F}_{9} \mathrm{~N}_{2} \mathrm{O}$ : C, 35.10; H, 2.06; N, 8.19. Found: C, 34.71; H, 2.00; N, 7.87.

2-Ethoxy-5-(perfluorohexyl)pyrimidine (11b). Yield 75\%; mp 26-27 ${ }^{\circ} \mathrm{C} ;{ }^{1} \mathrm{H}$ NMR $\left(\mathrm{CDCl}_{3}\right) \delta$ $1.47(\mathrm{t}, \mathrm{J}=7 \mathrm{~Hz}, 3 \mathrm{H}), 4.53(\mathrm{q}, \mathrm{J}=7 \mathrm{~Hz}, 2 \mathrm{H}), 8.70(\mathrm{~s}, 2 \mathrm{H}) ;{ }^{19} \mathrm{~F} \mathrm{NMR}\left(\mathrm{CDCl}_{3}\right) \delta 35.8(2 \mathrm{~F}), 39.1$ (2F), 39.9 (2F), 40.6 (2F), 50.7 (2F), 81.1 (3F). EI-MS m/z 145 (100), 442 (25, $\left.\mathrm{M}^{+}\right)$. Analysis. Calcd. for $\mathrm{C}_{12} \mathrm{H}_{7} \mathrm{~F}_{13} \mathrm{~N}_{2} \mathrm{O}$ : C, 32.60; H, 1.60; N, 6.34. Found: C, 32.57; H, 1.52; N, 6.23.

\section{5-(Perfluoroalkyl)pyrimidin-2(1H)ones 12a,b}

Hydrolysis of $\mathbf{1 1}$ to give $\mathbf{1 2}$ was conducted by using the procedure for $\mathbf{6}$ described above.

5-(Perfluorobutyl)pyrimidin-2(1H)one (12a). Yield 84\%; mp 205-206 ${ }^{\circ} \mathrm{C}$; ${ }^{1} \mathrm{H}$ NMR (DMSO$\left.d_{6}\right) \delta 8.65$ (s, 2H), 12.5 (bs, exchangeable with $\mathrm{D}_{2} \mathrm{O}, 1 \mathrm{H}$ ); ${ }^{19} \mathrm{~F}$ NMR (DMSO- $d_{6}$ ) $\delta 37.3(2 \mathrm{~F}), 40.1$ (2F), 52.9 (2F), 82.1 (3F). EI-MS m/z 145 (100), 314 (30, $\mathrm{M}^{+}$). Analysis. Calcd. for $\mathrm{C}_{8} \mathrm{H}_{3} \mathrm{~F}_{9} \mathrm{~N}_{2} \mathrm{O}$ : C, 30.59; H, 0.96; N, 8.92. Found: C, 30.43; H, 1.06; N, 8.87.

5-(Perfluorohexyl)pyrimidin-2(1H)one (12b). Yield 93\%; mp 209-211 ${ }^{\circ} \mathrm{C} ;{ }^{1} \mathrm{H}$ NMR (DMSO$\left.d_{6}\right) \delta 8.60$ (s, 2H), 12.9 (bs, exchangeable with $\mathrm{D}_{2} \mathrm{O}, 1 \mathrm{H}$ ); ${ }^{19} \mathrm{~F}$ NMR (DMSO- $d_{6}$ ) $\delta 36.9$ (2F), 40.2 (2F), 41.1 (2F), 41.5 (2F), 53.2 (2F), 82.3 (3F). EI-MS m/z 145 (100), 414 (20, $\mathrm{M}^{+}$). Analysis. Calcd. for $\mathrm{C}_{10} \mathrm{H}_{3} \mathrm{~F}_{13} \mathrm{~N}_{2} \mathrm{O}$ : C, 29.00; H, 0.73; N, 6.76. Found: C, 29.19; H, 0.67; N, 6.74.

\section{Acknowledgments}

This research was possible thanks to the financial assistance by the CHANNEL program of the government of Egypt and the Educational Aid Program of DuPont.

\section{References}

1. Curreri, A. R.; Ansfield, R. J.; McIver, F. H.; Waisman, H. A.; Hedelberger, C. Cancer Res. 1958, 18, 478.

2. Chaudhary, N. K.; Montag, B. J.; Heidelberger, C. Cancer Res. 1958, 18, 318. 
3. De Clercq, E. Pyrimidine Nucleoside Analogues as Antiviral Agents; In Targets for the Design of Antiviral Agents; De Clercq, E.; Walker, R. T. Eds, Plenum: New York, 1984; pp 203-230.

4. Andres, P.; Marhold, A. J. Fluorine Chem. 1996, 77, 93; and references cited therein.

5. Nishida, M.; Fujii, S.; Kimoto, H.; Hayakawa, Y. J. Fluorine Chem. 1993, 63, 43.

6. Schwarz, B.; Cech, D.; Reefschläger, J. J. Prakt. Chem. 1984, 326, 985.

7. Médebielle, M.; Pinson, J.; Savéant, J. M. Tetrahedron Lett. 1992, 33, 7351.

8. Médebielle, M.; Oturan, M. A.; Pinson, J.; Savéant, J. M. J. Org. Chem. 1996, 61, 1331.

9. Cech, D.; Wohlfeil, R.; Etzold, G. Nucleic Acids Res. 1975, 2, 2183.

10. Cech, D.; Herrmann, G.; Staske, R.; Langen, P.; Preussel, B. J. Prakt. Chem. 1979, 321, 488.

11. Kobayashi, Y.; Yamamoto, K.; Asai, T.; Nakano, M.; Kumadaki, I. J. Chem. Soc., Perkin Trans. 1, 1980, 2755.

12. Strekowski, L.; Ismail, M. A.; Zoorob, H. H. Heterocycl. Commun. 1998, 4, 399.

13. Strekowski, L.; Lin, S. Y.; Lee, H.; Wydra, R. L.; Kiselyov, A. S. Heterocycl. Commun. 1997, 3, 109.

14. Strekowski, L.; Lin, S. Y.; Lee, H.; Mason, J. C. Tetrahedron Lett. 1996, 37, 4655.

15. For a review, see: Kiselyov, A. S.; Strekowski, L. Org. Prep. Proced. Int. 1996, 28, 289.

16. For a review, see: Kobayashi, Y.; Kumadaki, I. Acc. Chem. Res. 1978, 11,197.

17. Santi, D. V.; Sakai, T. T. Biochemistry 1971, 10, 3598.

18. Simmons, H. E.; Wiley, D. W. J. Am. Chem. Soc. 1960, 82, 2288.

19. Kumar, P.; Wiebe, L. Nucleosides and Nucleotides 1990, 9, 861.

20. Crosby, D.; Berthold, R. J. Org. Chem. 1960, 25, 1916.

21. Brown, D.; Lyall, J. Aust. J. Chem. 1964, 17, 794.

22. Brown, D.; Lee, T. Aust. J. Chem. 1968, 21, 243. 